\title{
Testing of palm fibre as reinforcement material in polyester composites
}

\author{
V. V. S. Prasad ${ }^{1}$, D. N. Rao ${ }^{2}$, K. N. S. $\operatorname{Suman}^{2}$ \\ \& N. R. M. R. Bhargava ${ }^{3}$ \\ ${ }^{I}$ Department of Marine Engineering, Andhra University, \\ Visakhapatnam-53003, India \\ ${ }^{2}$ Department of Mechanical Engineering, Andhra University, \\ Visakhapatnam-53003, India \\ ${ }^{3}$ Department of Metallurgical Engineering, Andhra University, \\ Visakhapatnam-53003, India
}

\begin{abstract}
In this present work, palm fibre is incorporated in a polyester resin matrix to form unidirectional reinforced composites and bi-directional composites. Samples of different fibre volume fractions are fabricated and specimens with $0^{\circ}$, $45^{\circ}$ and $90^{\circ}$ fibre orientations are prepared. The specimens are tested on a universal testing machine applying tensile force. The tensile strength is measured as a function of fibre volume fraction. These properties follow "Rule of mixtures" relationship, with the volume fraction of palm. Because of the low density of natural fibers and high electrical resistance, these composites are more suitable for electrical and mechanical applications.

Keywords: palm fibre, hand lay up technique, mechanical and electrical properties.
\end{abstract}

\section{Introduction}

There is a great interest in the development of new materials which enhance optimal utilization of natural resources, and particularly of renewable resources. Natural fibres such as palm, jute, coir, banana, sisal etc., belong to this category. These fibres are abundantly available in developing countries, particularly in 
India and some places of South Africa [1]. The cotton polymer composite made contributions during the II world war [2,3]. As fibre reinforced plastics, it was first used by the military for radar domes on aircraft. During that period, bearings for ships were made of cotton-phenolic systems; also, brake linings of plywood-phenolic trainer wings and fuselages of British Mosquito bombers, and more than a thousand other items were made. During 1942, the Goodyear Aerospace Corporation for use in aircraft fuel cell produced backing sheet materials made of cotton fabric-polyester. It is recently reported [4] that cotton fabric reinforced phenolic resin composites have been used as bearings in place of phosphor bronze in the roll necks of steel and non-ferrous rolling mills. This resulted in energy savings up to $25 \%$.

Reddy, et al $[6,7]$ studied on fabrication, testing, damage characterization and feasibility of jute-polyester composites. Unprocessed jute yarn and fabric are used as fibers. Twisted jute yarn and fabric, which are semi finished raw materials and commercially produced widely in India, are selected for the work. General-purpose polyester is used as the matrix. Jute reinforced polyester laminates are prepared using 'Hand lay-up' technique to simulate practical production methods. Results indicate that there exists definite correlation between the tensile strength or elastic modulus and fiber volume fraction of the composite and with variation in fiber orientation in the composite.

One of the earliest natural fibre-polymer composites are investigated by Paramasivan and Abdulkalam [5] by incorporating sisal fibres and epoxy matrix. The fabrication process attempted by them includes winding and lamination. It is found that the fabrication of these composites is fairly easy and cost of production is quite low. Winding of cylinders with longitudinal or helical and hoop reinforcements is successfully carried out. Tensile strength of the sisalepoxy composites is found to be $250-300 \mathrm{Mpa}$, which is nearly half the strength of fibre glass-epoxy composites of the same composition. Because of the low density of sisal fibre, however, the specific strength of sisal composites is comparable with that of glass composites. The unidirectional modulus of sisalepoxy composites is found to be about $8.5 \mathrm{Gpa}$. This study indicates the feasibility of developing composites incorporating one of the abundantly available natural fibres, to be used in the field of consumer goods, low-cost housing and civil structures.

Lakkad and Patel [8] compared the values of ultimate tensile and compressive strength and young's modulus of elasticity of bamboo specimens with those of mild steel and glass reinforced plastics. But they have not specified the speciename of the bamboo specimens tested. There are more than 500 species of bamboo available in India and each has different mechanical properties.

Extensive literature is available on the production and mechanical behavior of composites obtained by reinforcing epoxy with fibre of glass, boron, carbon silicon carbide etc. Many researchers in the past have developed composites with natural fibres such as sisal henequen, jute, banana, cotton, etc., but the work on the palm reinforced plastic composites and palm reinforced oriented plastic composites are not available in the literature. 


\section{Fabrication of palm reinforced plastic composites}

Palm fibre is extracted from the leaf stem of the palm tree, which is not optimally used. Initially the cut stem from the plant is collected in a heap, and the stem is kept wet by spraying water for 48 hours for free release of the husk. Later by tapping lightly with wooden hammer, on the stem the fibre is separated in two forms as coarse fibre (i.e. $150 \mu \mathrm{m}-1500 \mu \mathrm{m}$ ), length up to $500 \mathrm{~mm}$ and fine fibre (i.e. $75 \mu \mathrm{m}-150 \mu \mathrm{m}$ ) length up to $70 \mathrm{~mm}$ The fibres are flexible compared to the coarser fibres and segregated in the form of bundles. A rectangular thick tapered plastic plate of size $(200 \times 50) \mathrm{cm}^{2}$ is used as a mould for making the composite by using "Hand- lay-up technique". Acetylene is used as a cleaning agent for cleaning the casting surface of the mould, a releasing agent polyvinyl alcohol is used for easy removal of the casting. After thoroughly mixing the resin with hardener, it is applied over the entire sheet using a soft brush and a coat of wax is applied on this resin layer. The finer fibres are inserted in the wax placing them parallel to the longer edges of the mould plate, and brushing is done smoothly so that resin spreads through the yarn. Care is also been taken to see that the yarns are not being displaced from respective positions after brushing. This process is repeated till all the palm fibres are wet properly.

The laminates are cured at room temperature for 24 hours. Laminates with approximately $10 \%, 20 \%, 30 \%$ and $40 \%$ of the fibre volume fraction are prepared as shown Fig 2. For the laminates with volume fraction $50 \%$ above it is

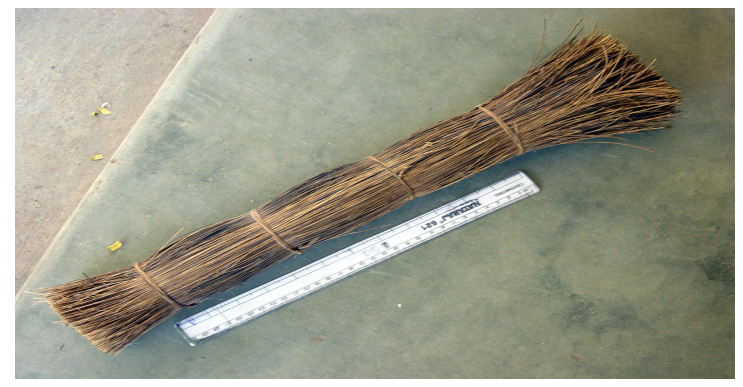

Figure 1: Palm fibre (finer type) bundle.

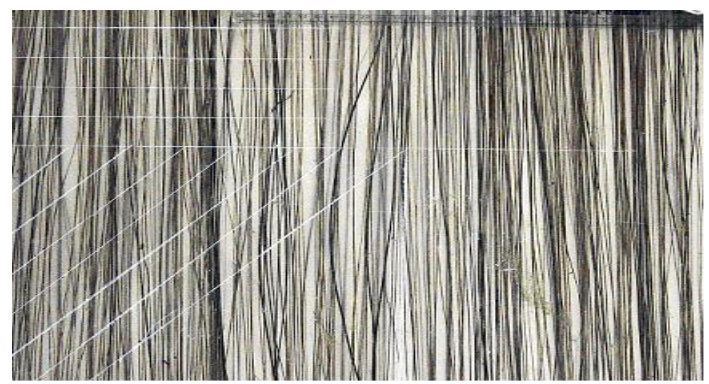

Figure 2: $\quad$ Palm reinforced plastic laminate. 
observed that wetting of the fibre is not proper and there is no much improvement in strength.

To fabricate the bi-directional composite, the second layer of palm is placed perpendicular to the first layer and the above process is repeated till the resin spreads over the entire surface. Fig. 3 shows the bi-directional composites.

Oriented fibre composites are prepared by placing second layer of palm at different angles of $15^{\circ}, 30^{\circ}$ and $45^{\circ}$ for each composite. Figs.4-6, depict the various orientations respectively.

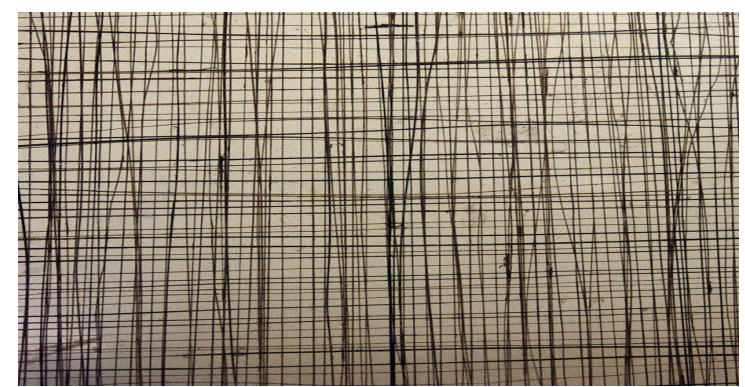

Figure 3: Palm bi-directional composites.

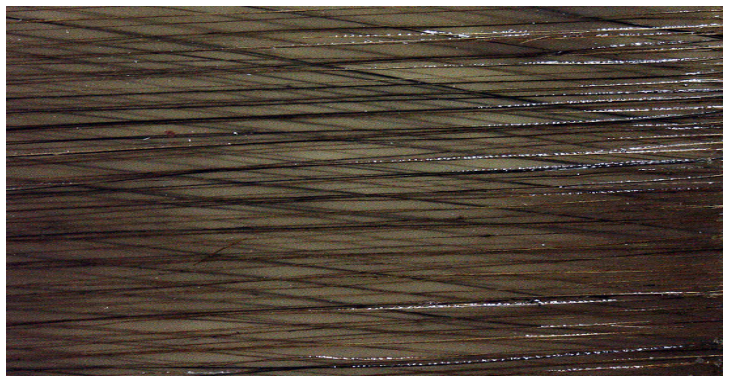

Figure 4: $\quad$ Laminates with $15^{\circ}$ fibre orientation with a fibre volume of $20 \%$.

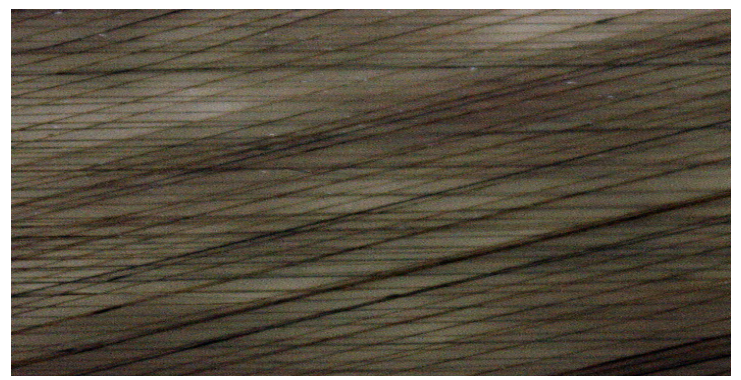

Figure 5: $\quad$ Laminates with $30^{\circ}$ fibre orientation with a fibre volume of $20 \%$. 


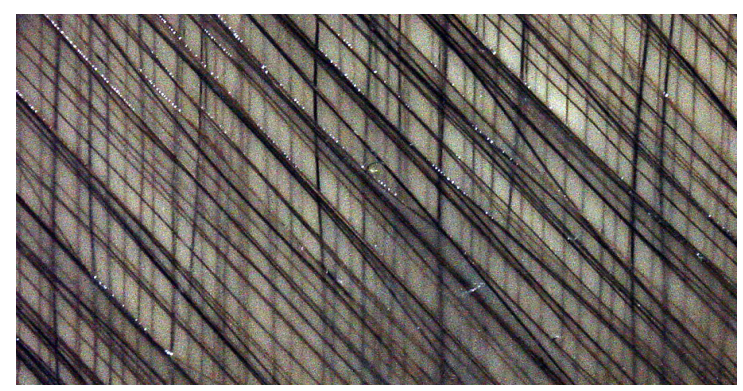

Figure 6: $\quad$ Laminates with $45^{\circ}$ fibre orientation with a fibre volume of $20 \%$.

\section{Experimental procedure}

\subsection{Tensile test}

As per ASTM standards the specimen are prepared to the required size of $250 \mathrm{~mm} \times 25.5 \mathrm{mmx} 4 \mathrm{~mm}$ with $0^{\circ}, 45^{\circ} 90^{\circ}$ of fibre orientation. The standard specimens are marked with marking scriber and samples are cut to size using power band saw. PFRP samples are tested on tensile testing machine; UNITEK95100 under a load of $25 \mathrm{KN}$ and with a cross head speed of $20 \mathrm{~mm} / \mathrm{min}$. The specimens are held by flat graved grips. To avoid slipping of grippers during load application, the ends of specimens are made rough by filing. The breaking loads and displacements at various loads are measured. The observing results are presented in figs. 7, 9 and 10 for different percentages of volume fraction and orientations of fibre.

\subsection{Electrical test}

The present test is designed to measure the leakage current between two points. Leakage test is conducted on Cascade transformer, $100 \mathrm{kVA}, 500 \mathrm{kV}, 200 \mu \mathrm{A}$ and an ammeter is connected to measure the leakage current. Resistance is calculated using the Kirchoff's law (V=I R) at constant voltage. Specimens in the normal and soaked in sea water for $12 \mathrm{hrs}$, are tested for breakdown voltage, at the High Voltage Laboratory, Department of Electrical Engineering, Jawaharlal Nehru Technical University, Kakinada, Andhra Pradesh, India.

\section{Results}

From Fig. 7, it is observed that the tensile strength of the composites increases with increase in the fibre volume fraction. Fibres are the main load carrying agents in composites and as the number of load carrying elements increases in a material, its strength increases. The composite tensile strength decreases with increasing the orientation of the fibre from $0^{\circ}$ to $90^{\circ}$. 


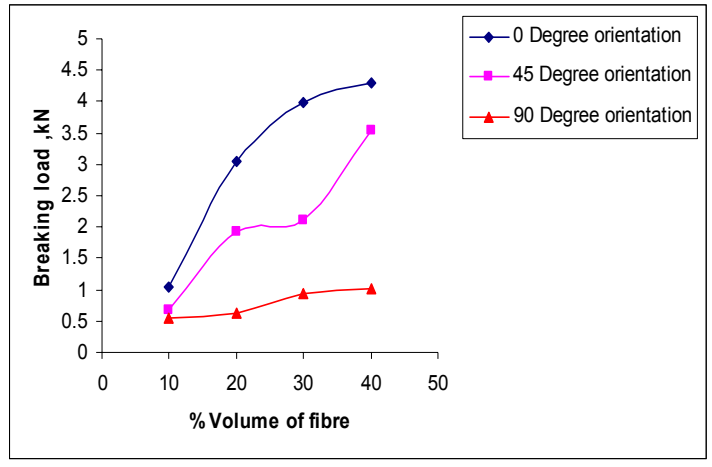

Figure 7: Effect of \% volume and orientation of palm fibre on breaking load for uni-directional composite.

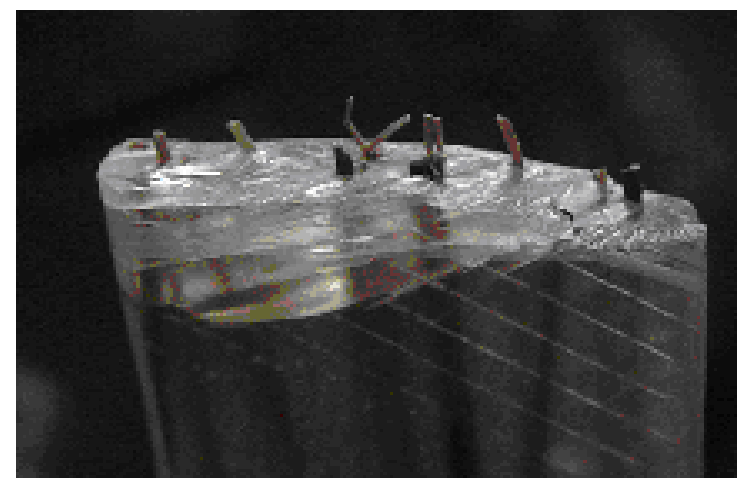

Figure 8: Fractured surface of 10 vol \% palm fibre reinforced polymer composite $10 \mathrm{X}$.

Figure 8 shows the fractured surface of the 10 vol \% palm fibre reinforced composite. Matrix is found to be deformed to a lesser extent while fibres are protruding from the surface. It shows that fibres have been pulled away from matrix indicating poor bonding at the fibre- matrix interface. This effect is much more pronounced at higher percentages of the fibre and has resulted in lower breaking load values compared to the theoretical calculations of rule of mixtures.

Figure 9 shows the effect of fibre volumes and the fibre orientation on the breaking load of bi-directional composites. Since the composite is made bidirectional, breaking load values for the fibre orientations of $0^{\circ}$ and $90^{\circ}$ have shown similar values at all the fibre contents. These values are found to increase with increasing fibre contents. Composites with fibre orientations of $45^{\circ}$ have shown a similar trend but have shown lesser strength values. These results are self explanatory as the strength of the fibre in the warp direction is more than that of in the weft direction. 


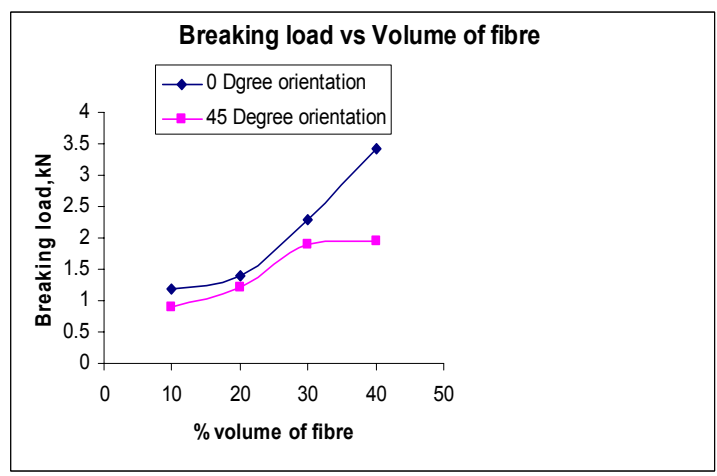

Figure 9: Effect of palm fibre volume and orientation on breaking load of bidirectional composite.

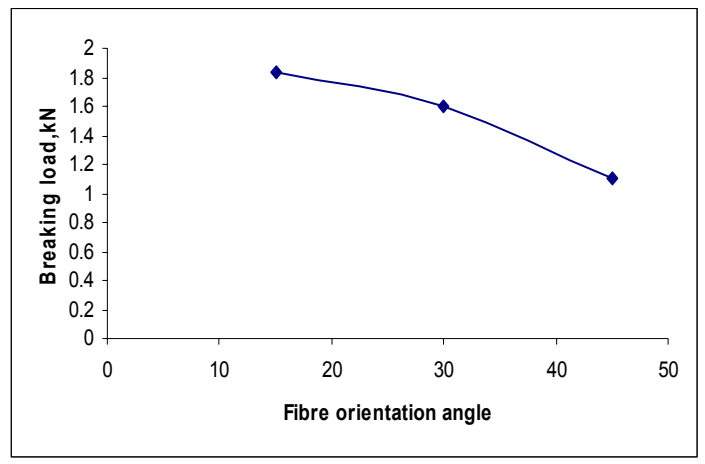

Figure 10: Effect of palm fibre volume and orientation on breaking load of palm oriented composites.

Figure 10 shows the effect of fibre orientation angle on the tensile strength of the composites. As the orientation angle increases the tensile strength drops to a minimum at the maximum weft of $45^{\circ}$.

Figure 11 shows the effect of palm fibres volume on the leakage current. Leakage current found to be increasing with increased fibre volumes initially and almost stabilizes at higher contents. From the literature it is found that presence of voids and air pockets enhance the leakage currents. Since palm is natural one and is also in the thoroughly dried condition, sufficient voids are readily present in it. This might have lead to the increased leakage currents in the reinforced composite. As the fibre volume increases, the presence of these discontinuities also increases which might have lead to the increased leakage currents. During processing increased fiber volumes enhance the chances of void presence due to practical problems. This might have further accentuated the leakage voltage at higher volumes of the fibre. 


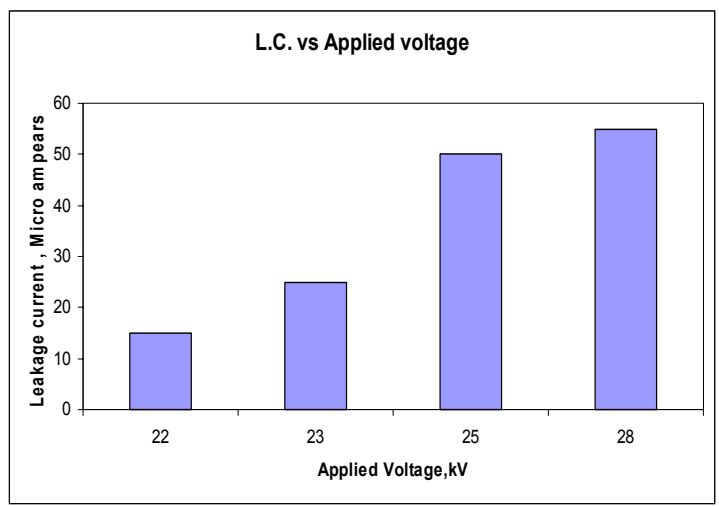

Figure 11: Effect of palm fibre volume on leakage current.

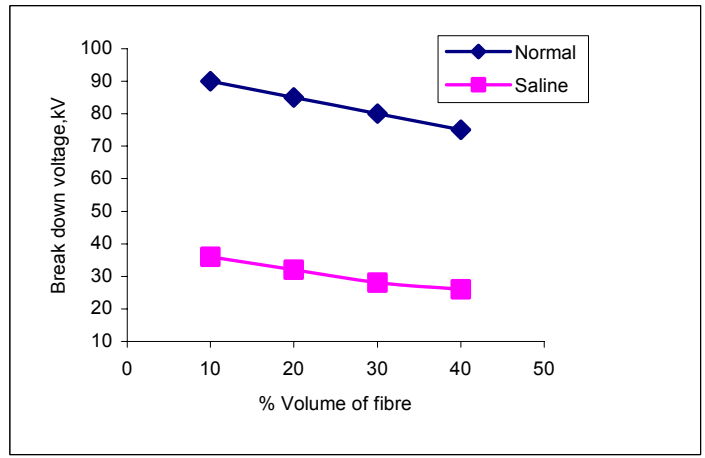

Figure 12: Effect of fibre volume on break down voltage.

Figure 12 shows the effect of fibre volume on breakdown voltage. Specimens soaked in the saline water have shown drastic drop in the breakdown voltages compared to the normal samples. A similar trend of drop in breakdown voltage with increasing fibre volumes has been observed with both the conditions of normal and seawater soaked ones. Since the presence of voids, impurities and the moisture decreases the breakdown voltage, the above discussion holds good for this behaviour as well. Presence of moisture has dropped the values further.

\section{Conclusions}

1. Palm fibre can be used as reinforcement and filler in the polymer based composites.

2. It shows a conventional behaviour in mechanical properties depicting higher breakdown strength values with increasing fibre volumes.

3. Composites with fibre orientation in the warp direction exhibit better mechanical properties than the weft direction ones. 
4. Leakage current increases with increasing fibre volumes.

5. Breakdown voltage decreases with increasing fibre volumes.

6. Saline water soaked samples exhibit poor breakdown voltage compared to the normal ones.

\section{References}

[1] Satyanarayana, K.G., Kulkarni, G.Sukumaran, K., Pillai, S.G.K. Cheriyan, K.A. and Rohatgi, P.K., "on the possibility of using natural fiber polymer composites". Proc. First International Conference on Composite Structures, 16-18(Sept., 1981), ed. 1.H.Marshall. Applied Science publishers, London, pp.618-623.

[2] Piggot, M.R., "Load Bearing Fiber Composites". Pergamon press, Oxford, 1980.

[3] Lubin, G. (ed), "Hand Book of Composites". Van Nostrand Reinhold, New York 1982.

[4] "Save energy - Save money - composite news. Composites", 10 (April 1979) pp.61.

[5] Parmasivam, T and Abdulkalam, A.P.J., "On the study of natural fiber composites” Fiber Science and Technology I (1974) pp. 85-88.

[6] Govardhan Reddy, B., Rao, D.N., Bhargava, N.R.M.R. Prasad, V.V.S,. "Damage Mechanism under tensile loading of continuous jute reinforced polyester composites" Proc. Third International conference on 'Advances in composites' ADCOMP-2000, August 2000, Bangalore, India. pp.24-26.

[7] Govardhan Reddy, B., Rao, D.N., and Rao, R.N.S. “Jute-reinforced polyester composites - A study of properties", Proc. Of $11^{\text {th }}$ AGM, Materials Research Society of India, India, July 2000.

[8] Lakkad, S. C. and Patel, J. M., "Mechanical Properties of Bamboo, a Natural Composite," Fibre Science and Technology, Vol. 14, (1980-81) pp. 319-322. 